\title{
Barrier screens: a method to sample blood-fed and host-seeking exophilic mosquitoes
}

Thomas R Burkot ${ }^{1,2^{*}}$, Tanya L Russell ${ }^{1}$, Lisa J Reimer ${ }^{3,4}$, Hugo Bugoro ${ }^{5}$, Nigel W Beebe ${ }^{6,7}$, Robert D Cooper ${ }^{8}$, Supraman Sukawati ${ }^{9}$, Frank H Collins ${ }^{10}$ and Neil F Lobo ${ }^{10}$

\begin{abstract}
Background: Determining the proportion of blood meals on humans by outdoor-feeding and resting mosquitoes is challenging. This is largely due to the difficulty of finding an adequate and unbiased sample of resting, engorged mosquitoes to enable the identification of host blood meal sources. This is particularly difficult in the south-west Pacific countries of Indonesia, the Solomon Islands and Papua New Guinea where thick vegetation constitutes the primary resting sites for the exophilic mosquitoes that are the primary malaria and filariasis vectors.

Methods: Barrier screens of shade-cloth netting attached to bamboo poles were constructed between villages and likely areas where mosquitoes might seek blood meals or rest. Flying mosquitoes, obstructed by the barrier screens, would temporarily stop and could then be captured by aspiration at hourly intervals throughout the night.

Results: In the three countries where this method was evaluated, blood-fed females of Anopheles farauti, Anopheles bancroftii, Anopheles longirostris, Anopheles sundaicus, Anopheles vagus, Anopheles kochi, Anopheles annularis,

Anopheles tessellatus, Culex vishnui, Culex quinquefasciatus and Mansonia spp were collected while resting on the barrier screens. In addition, female Anopheles punctulatus and Armigeres spp as well as male An. farauti, Cx. vishnui, Cx. quinquefasciatus and Aedes species were similarly captured.
\end{abstract}

Conclusions: Building barrier screens as temporary resting sites in areas where mosquitoes were likely to fly was an extremely time-effective method for collecting an unbiased representative sample of engorged mosquitoes for determining the human blood index.

Keywords: Mosquito sampling, Human blood index, Barrier screen trap, Exophily

\section{Background}

The importance of a mosquito species as a vector of human disease is determined by multiple parameters including the human blood index (HBI). The HBI is the proportion of blood-fed, resting mosquitoes that contain human blood in their stomachs compared to the total number of mosquitoes which feed on any host species [1]. Accurate estimates of the HBI for a species depends on collecting an unbiased sample of resting, blood-fed mosquitoes of that species [2]. For highly endophilic species that rest for prolonged periods inside houses, it is relatively easy and straightforward to collect large

\footnotetext{
* Correspondence: tom.burkot@jcu.edu.au

${ }^{1} J a m e s$ Cook University, Queensland Tropical Health Alliance, Cairns, QLD 4870, Australia

${ }^{2}$ Centers for Disease Control and Prevention, Division of Parasitic Diseases and Malaria, Atlanta, GA 30333, USA

Full list of author information is available at the end of the article
}

numbers of engorged mosquitoes inside houses. However, HBI estimates based solely on collections of indoor-resting mosquitoes, even for endophilic mosquitoes, are biased towards human-fed mosquitoes, as such collections ignore the portion of the population that will have fed outdoors and be more likely to have fed on other available host species. Accurate HBI estimates require unbiased samples of the entire mosquito population that has recently fed (i.e., those that feed and rest inside houses as well as those that feed and rest outside).

Exophilic mosquito species are common throughout the malaria-endemic world [3-5]. For exophilic vectors, capturing an adequate and representative sample of blood-fed specimens is even more challenging as they tend to be dispersed over large areas and utilize a large number of potential resting sites. The challenge is magnified when the number of adult vectors is limited, as

\section{Biomed Central}


finding engorged resting mosquitoes outdoors requires considerable time and effort to acquire even a small sample of blood-fed mosquitoes [2]. To enhance the prospects of finding blood-fed mosquitoes outside houses, artificial resting sites, such as clay pots and resting boxes may be provided or pits dug to attract engorged females [6]. However, artificial resting sites may harbour relatively few blood-fed, resting mosquitoes since the artificial sites provided must compete with the greater number of available natural resting sites $[7,8]$.

In response to biases associated with sampling bloodfed, resting mosquitoes, a novel sampling tool was designed based on a hypothesis that engorged mosquitoes might be intercepted and captured when transiting between blood feeding and resting sites. This hypothesis was based on observations by Giglioli [9] and Gillies and Wilkes [10] about the flight patterns of anophelines. Giglioli [9] reported that Anopheles melas entered villages in corridors and at altitudes less than five feet $(1.53 \mathrm{~m})$ and that their flight could be diverted by fences. Gillies and Wilkes [10] confirmed that most mosquitoes fly at low altitudes when crossing open terrain although anophelines will modify the height of their flight when they encounter obstacles. It followed that obstacles could be constructed, not to divert flying mosquitoes, but to impede their flight sufficiently to allow capture. For blood-laden mosquitoes, any structure encountered between the host and sites for resting might be sufficient to provide a temporary rest stop for the mosquito. Barrier screens constructed of durable mesh material (shade cloth) are inexpensive, easily constructed, and easily searched for resting mosquitoes from which they can be collected. As an artificially constructed resting site or flight barrier, a screen provides an additional significant advantage over a solid structure in that the mesh is permeable and would permit mosquitoes to follow both visual and olfactory cues to sources of blood meals, oviposition and resting sites. Upon encountering the barrier screen, mosquitoes might then stop, thereby facilitating their discovery and capture. Placement of a barrier screen between likely oviposition/resting sites and potential blood meal sources would enable the capture of both blood-fed mosquitoes seeking a resting site to develop their eggs, as well as recently emerged mosquitoes that have completed egg-laying and/or are searching for a blood meal. Comparisons of blood meal, parity and gravid status of mosquitoes captured on each side of such a screen would provide insight into directional and temporal behaviour patterns.

This initial evaluation of barrier screens as a novel sampling tool to collect exophilic mosquitoes was conducted in three countries in the southwest Pacific (Indonesia, the Solomon Islands and Papua New Guinea). The current paper presents the results of pilot studies to optimise the use and placement of barrier screens and to describe the physiological state of the mosquitoes captured. In this region the primary malaria and lymphatic filariasis vectors are exophilic, including An. sundaicus, An. vagus, An. kochi, An.s annularis, An. tessellatus and the members of the An. punctulatus group [3,11,12]. Previous HBI estimates for the $A n$. punctulatus group required years of effort to collect a sufficient number of blood-fed, resting specimens for blood source identification. This was primarily due to the difficulty of finding mosquitoes resting amongst the thick vegetation that serve as the usual resting sites for these mosquitoes $[6,13]$. Thus, only a limited number of studies have documented the host blood meal sources in the members of this group in the Solomon Islands [14-16] and Papua New Guinea [13,16-22], the most recent of which was published 16 years ago.

\section{Methods}

\section{Barrier screens}

The barrier screens were constructed from ether polyvinylchloride-coated polyester or polyethylene shade cloth (70\% shading) netting. The shade cloth was obtained either from the manufacturer [23] or local hardware stores. The $2 \mathrm{~m}$-high barrier screens were constructed by securing the shade cloth to wooden or bamboo poles at $2 \mathrm{~m}$ intervals (Figure 1A,D) with zip-ties or polyester cord (Figure 1B,E, respectively) and the barrier screens searched during the night for resting mosquitoes, which were then captured by aspirations (Figure 1C,F). The efficacy of the barrier screens for facilitating the collection of exophilic mosquitoes was evaluated in sites in three countries.

\section{Mosquito sampling}

The experiments involved sampling mosquitoes from the barrier screens or by using human landing catch (HLC) in all three sites. All mosquito catches were conducted between $1800 \mathrm{~h}$ and $0600 \mathrm{~h}$, and the catches for each hourly interval were stored in separate collection cups. Regarding sampling resting mosquitoes from the barrier screens, this process was conducted by manually searching the barrier screen and collecting any resting mosquitoes with a mouth aspirator (Figure $1 \mathrm{C}$ and F). Each side of the barrier screen was searched for approximately $20 \mathrm{mins}$ each hour. The catches for each side of the barrier screen were stored in separate collection cups. To collect host-seeking mosquitoes HLC were conducted outdoors. This involved volunteers sitting with their legs and feet exposed and catching mosquitoes with a mouth aspirator that were attracted and seeking a blood meal; HLC collections were also made hourly from $1800 \mathrm{~h}$ to $0600 \mathrm{~h}$ [6]. All mosquitoes were morphologically identified to sex and species then 


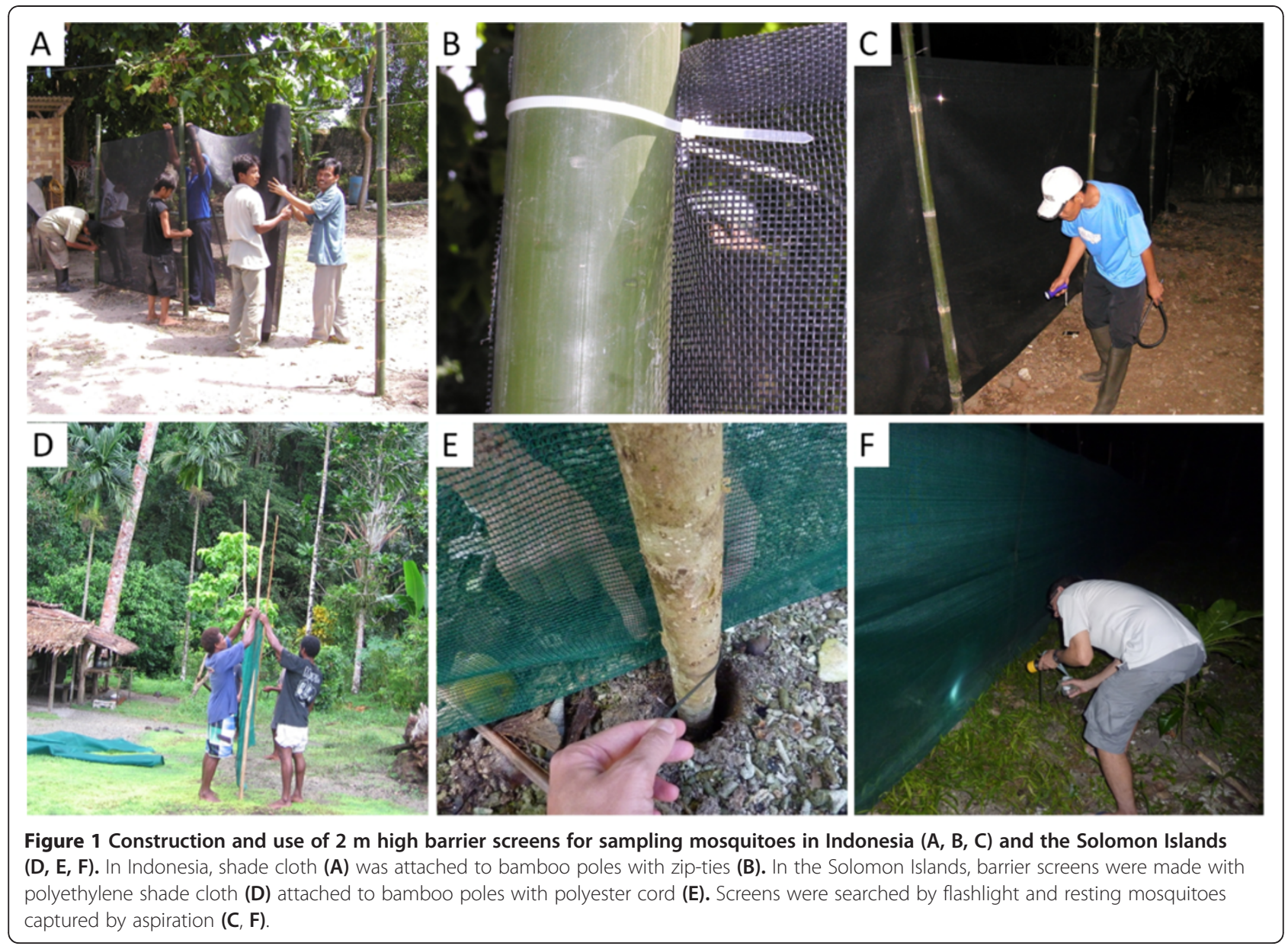

visually classified as being unfed, partially fed, fully fed or gravid. The morphological keys used to identify the mosquito specimens were O'Connor and Soepanto [24] in Indonesia, Belkin [11] in the Solomon Islands, and Lee [25] in Papua New Guinea. Species identifications were confirmed by molecular analyses (see Laboratory analyses below). The collection details for each mosquito were recorded, including trap type and hour of capture.

\section{Indonesia}

A $32 \mathrm{~m}$ long barrier screen of polyvinylchloride-coated polyester shade cloth was constructed (Figure 1A,B) and evaluated in Sukaraja village in the Lampung District (Rajabasa subdistrict) of southern Sumatra in western Indonesia between 25 August and 1 September 2010. Mosquitoes were collected from the barrier screen over eight nights. The location of mosquitoes was recorded for each mosquito (e.g., $<0.5 \mathrm{~m} ; 0.5$ to $<1.0 \mathrm{~m}$; $>1 \mathrm{~m}$ above the ground). Domestic animals that might serve as potential host blood meal sources were recorded. After the mosquito processing was complete, unfed female anophelines were dissected for parity status.
Additionally, HLC was performed on the same nights as the barrier screen collections at 6 outdoor stations.

\section{Solomon Islands}

In Haleta village on Big Nggela Island, Central Province, Solomon Islands, the barrier screen experiments were refined to intercept mosquitoes either seeking blood meals or searching for resting sites. The layout of Haleta village provided an ideal scenario to test a hypothesis that barrier screens could intercept mosquitoes entering the village searching for a blood meal after having just emerged or after having just laid eggs, as well as intercepting blood-fed mosquitoes leaving the village and seeking a resting site to develop their eggs. Previously, larval surveys identified a single dominant breeding site, a swamp formed by the blockage of a stream by a sandbar. This swamp was surrounded by thick vegetation, suitable resting sites for blood-fed mosquitoes, whereas in the village where there was little or no vegetation that might serve as resting sites. A barrier screen of approximately $20 \mathrm{~m}$ was constructed between the swamp and the village houses (Figure 2A). Resting mosquitoes were 


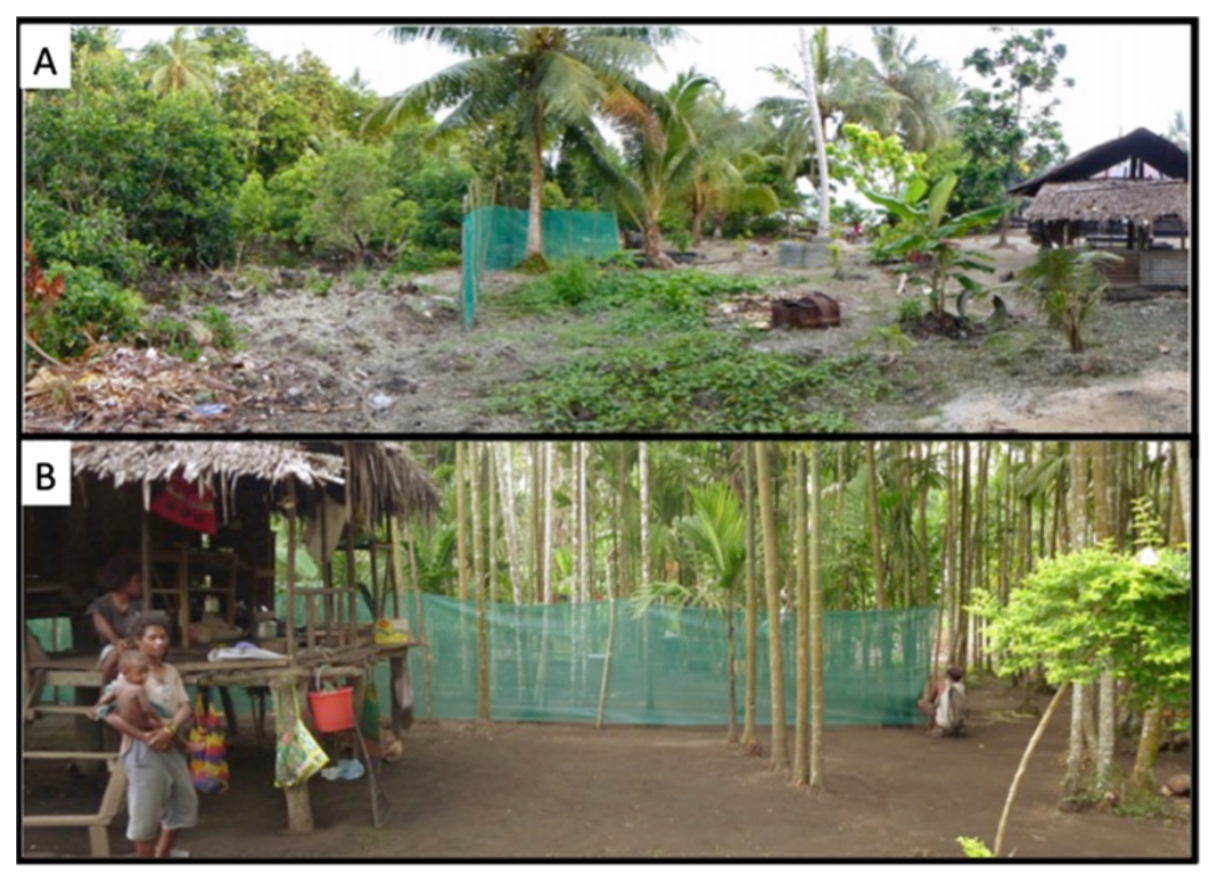

Figure 2 Barrier screens were constructed between village houses and potential resting and/or oviposition sites, as shown in Haleta village, Solomon Islands (A) and Mirap village, Papua New Guinea (B). Potential resting sites among the vegetation and the primary oviposition site (a brackish water swamp) can be seen to the right of the barrier screen while village houses and animal pens (seen to the left of the barrier screen) provide potential blood meals.

collected from the barrier screen for 14 consecutive nights in November 2011.

Concurrent with barrier screen collections, HLC were conducted from $1800 \mathrm{~h}$ to $0600 \mathrm{~h}$ at 6 outdoor stations. Mosquitoes were held in individual containers by hour of collection and numbers recorded.

\section{Papua New Guinea}

A $40 \mathrm{~m}$ barrier screen was similarly constructed between houses and the surrounding vegetation in Mirap village, Madang Province (Figure 2B). The barrier screen was constructed in two 20-m segments for ease of set-up and to allow a $1 \mathrm{~m}$ gap for access to the surrounding bush along pre-existing trails. One pair of collectors checked the barrier screen for resting mosquitoes for 10 nights in June 2012. The mosquitoes were captured and stored by hour of collection and side of the barrier screen. Additionally, HLC was performed on the same nights as the barrier screen collections at 2 outdoor stations. Locations of screens and collectors were rotated throughout the village every two nights.

\section{Laboratory analyses}

Mosquito species identification of the An. punctulatus group was confirmed by PCR [26,27]. Sequencing of the ITS2 region [26] was also used to identify species in Indonesia. Host blood meal sources (human, pig, goat, and dog) for Anopheles mosquitoes were identified by
PCR using slight modifications of the Kent and Norris method [28]. The PCR reactions were made up of $0.4 \mu \mathrm{l}$ of each primer, $3.0 \mathrm{mM} \mathrm{MgCl}, 1.0 \mathrm{mM}$ dNTPs and 0.5 units of Taq polymerase and each reaction in a final volume of $25 \mu \mathrm{l}$.

\section{Statistical analyses}

For the Solomon Islands, statistical differences in the proportion of blood-fed to unfed mosquitoes resting on each side of the barrier screen were compared using a generalized linear model (GLM) with a binomial distribution and a categorical explanatory variable for screen side. The basis of the analysis was a binary dataset constructed with the total number of fed and unfed An. farauti collected on each side of the barrier screen by date. This analysis was conducted using $\mathrm{R}$ statistical software (ver.2.14.2).

\section{Ethics}

Ethical approval for the study was obtained from review boards relevant to each study site. For Indonesia approval was granted by the National Institute of Health, Research and Development, Indonesia. For the Solomon Islands, approval was granted by the National Health Research \& Ethics Committee (02-05-2011) as well as the James Cook University Human Research Ethics Committee (H4122). For Papua New Guinea, approval was granted by the PNG Institute of Medical Research 
Institutional Review Board (1116) and the PNG Medical Research Advisory Board (11.21). When the study commenced, permission was obtained from each person who volunteered to conduct HLC. After consenting, each volunteer signed an informed consent form stating their willingness to participate in the study.

\section{Results}

\section{Indonesia}

In southern Sumatra, $C x$. vishnui and $C x$. quinquefasciatus were the most abundantly collected species on the barrier screen ( $\mathrm{n}=1,057$ and 513, respectively), including 65 and 117 male $C x$. vishnui and $C x$. quinquefasciatus, respectively. Five species of anophelines were collected resting on the barrier screen. Anopheles sundaicus $(\mathrm{n}=26)$ was the most abundantly collected anopheline followed by An. vagus ( $\mathrm{n}=15)$, An. kochi $(\mathrm{n}=2)$ and $A n$. annularis and An. tessellatus (one each) (Table 1). The mean number of An. sundiacus collected per $10 \mathrm{~m}$ barrier screen per night was 1.0 and for An. vagus was 0.6. The mean nightly, human landing rate for An. sundiacus during the same period was $7.6 \mathrm{bites} / \mathrm{person} / \mathrm{night}$ $(\mathrm{b} / \mathrm{p} / \mathrm{n})$ and for An. vagus was $0.3 \mathrm{~b} / \mathrm{p} / \mathrm{n}$. The host seeking densities of An. tesselatus, An. kochi and An. annularis were negligible (Table 1).
When capturing mosquitoes on the barrier screen, it was observed that most (92\%) were captured resting $<1 \mathrm{~m}$ from the ground, and of these mosquitoes $74 \%$ were within $50 \mathrm{~cm}$ of the ground. All anophelines were collected $<1 \mathrm{~m}$ from the ground with $73 \%$ within $50 \mathrm{~cm}$ of the ground. Of the anophelines resting on the barrier screen, $25.5 \%(11 / 43)$ were blood fed, whereas only $6.5 \%$ (64/992) and 5.6\% (22/395) of Cx. vishnui and Cx. quinquefasciatus were blood fed. Gravid females represented $16.3 \%$ (7/43), $2.1 \%(21 / 992)$ and $8.9 \%(35 / 395)$ of anophelines, $C x$. vishnui and $C x$. quinquefasciatus collected on the barrier screen, respectively.

Goats, chickens, humans, dogs and cats were observed in the vicinity of the barrier screen. Only a small portion of the engorged females were tested for blood meal with PCR. All tested An. sundiacus contained dog blood $(\mathrm{n}=2)$ while all tested An. vagus had fed on goats $(\mathrm{n}=3)$. For Cx. quinquefasciatus, $25 \%, 50 \%$ and $25 \%$ of identified blood meals were on goats, dogs and humans, respectively $(\mathrm{n}=8$ successful PCR reactions where the blood meal host was identified, 5 additional blood meal tests could not be identified). For Cx. vishnui, 59\%, $20.5 \%$ and $20.5 \%$ of identified blood meals were on goats, dogs and humans, respectively ( $n=49$ successful PCR reactions, 3 could not be identified). The parity rate

Table 1 Tabular comparison of the density of host-seeking (HLC) and resting mosquitoes (barrier screen) caught in the three study sites: Indonesia, the Solomon Islands and Papua New Guinea

\begin{tabular}{|c|c|c|c|c|c|c|}
\hline \multirow[b]{3}{*}{ Species } & \multirow{3}{*}{$\begin{array}{c}\text { Host seeking } \\
\text { HLC b/p/n (total) } \\
\text { Female }\end{array}$} & \multicolumn{5}{|c|}{ Resting mosquitoes } \\
\hline & & \multicolumn{2}{|c|}{ Mean/10 m barrier screen (total) } & \multicolumn{3}{|c|}{ Abdominal status } \\
\hline & & Male & Female & Unfed\% (n) & Bloodfed\% (n) & Gravid\% (n) \\
\hline \multicolumn{7}{|l|}{ INDONESIA } \\
\hline An. sundiacus & $7.6(367)$ & $0.0(0)$ & $1.0(26)$ & $58.3(14)$ & $25.0(6)$ & $16.7(4)$ \\
\hline An. vagus & $0.3(15)$ & $0.0(0)$ & $0.6(15)$ & $53.5(8)$ & $26.7(4)$ & $20.0(3)$ \\
\hline An. tesselatus & $0.0(0)$ & $0.0(0)$ & $0.0(1)$ & $100.0(1)$ & $0.0(0)$ & $0.0(0)$ \\
\hline An. kochi & $0.0(2)$ & $0.0(0)$ & $0.1(2)$ & $50.0(1)$ & $50.0(1)$ & $0.0(0)$ \\
\hline An. annularis & $0.0(1)$ & $0.0(0)$ & $0.0(1)$ & $100.0(1)$ & $0.0(0)$ & $0.0(0)$ \\
\hline Cx. quinquefasciatus & NA & $4.2(117)$ & $15.5(396)$ & $85.6(338)$ & $5.6(22)$ & $8.9(35)$ \\
\hline CX. vishnui & NA & $2.5(65)$ & $38.8(992)$ & $91.4(907)$ & $6.5(64)$ & $2.1(21)$ \\
\hline \multicolumn{7}{|l|}{ SOLOMON ISLANDS } \\
\hline An. farauti s.s. & $16.5(1388)$ & $0.1(1)$ & $4.2(117)$ & $36.8(43)$ & $62.4(73)$ & $0.9(1)$ \\
\hline \multicolumn{7}{|l|}{ PAPUA NEW GUINEA } \\
\hline An. bancrofti & $0.0(0)$ & NA & $0.8(30)$ & $76.7(23)$ & $23.3(7)$ & $0.0(0)$ \\
\hline An. farauti s.s. & $20.6(412)$ & NA & $7.8(311)$ & $40.2(125)$ & $59.2(184)$ & $0.6(2)$ \\
\hline An. punctulatus & $0.0(0)$ & NA & $0.1(3)$ & $100.0(3)$ & $0.0(0)$ & $0.0(0)$ \\
\hline An. longirostris & $0.0(0)$ & NA & $0.1(2)$ & $50.0(1)$ & $50.0(1)$ & $0.0(0)$ \\
\hline Aedes spp. & NA & NA & $1.4(54)$ & $79.6(43)$ & $20.4(11)$ & $0.0(0)$ \\
\hline Culex spp. & NA & NA & $1.1(42)$ & $90.5(38)$ & $7.1(3)$ & $2.4(1)$ \\
\hline Mansonia spp. & NA & NA & $0.1(2)$ & $50.0(1)$ & $50.0(1)$ & $0.0(0)$ \\
\hline
\end{tabular}

The abdominal status of resting mosquitoes is noted.

$\mathrm{b} / \mathrm{p} / \mathrm{n}=$ bites/person/night; NA = Not Available. 
of the Anopheles spp was high at $86 \%(24 / 28)$ and also for the Culex spp at $71 \%(89 / 125)$.

\section{Solomon Islands}

A total of 117 female An. farauti were collected on the barrier screen (mean of $4.2 / 10 \mathrm{~m}$ barrier screen/per night; Table 1) with $84 \%(\mathrm{n}=98)$ captured on the village side and only $16 \%(n=19)$ on the side closest to the oviposition and resting sites. In addition, seven male Aedes spp as well as one Culex spp and one An. farauti male were collected on the barrier screen. Six of the seven male Aedes spp were captured between $0200 \mathrm{~h}$ and $0500 \mathrm{~h}$. The number of resting An. farauti peaked between $2000 \mathrm{~h}$ and $2100 \mathrm{~h}$ and then diminished and remained low for the remainder of the night (Figure 3B). During the 14 nights of the experiments, the mean nightly outdoor landing catch for An. farauti was $16.5 / \mathrm{p} / \mathrm{n}$ (Table 1). Peak human landing catches occurred between $1900 \mathrm{~h}$ and $2000 \mathrm{~h}$ (Figure 3A).

The percentage of blood-fed female An. farauti captured resting on the barrier screen was $62 \%(n=73)$, with $51 \%(n=60)$ of the total mosquitoes being fully engorged. There was a tendency for more blood-fed $A n$. farauti to be captured on the village side of the barrier screen (65\%) compared with the breeding-site side $(47 \%)$, but this difference was not statistically significant $(\beta=0.632, \mathrm{SE}=0.517, p=0.221)$. Only one gravid $A n$. farauti was collected on the barrier screen. The most abundant potential domestic host blood meal sources present in the village in the vicinity of the barrier screen were humans $(n=64)$, pigs $(n=11)$ and dogs, $(\mathrm{n}=4)$. The majority, 97\% $(\mathrm{n}=68)$, of blood-fed An. farauti contained human blood including $6 \%(\mathrm{n}=4)$ having mixed feeds on humans and pigs. Only 3\% $(\mathrm{n}=2)$ of $A n$. farauti had fed solely on pigs.

\section{Papua New Guinea}

A total of 449 female mosquitoes were collected from the barrier screen with $50 \%$ from the village side. Across all species, of those collected from the village side, 54\% were blood fed $(n=144)$ whereas only $34 \%$ were blood fed $(\mathrm{n}=63)$ on the opposite side (furthest from the village). Anopheles farauti was the predominant species, comprising $69 \%$ of the catch $(\mathrm{n}=311)$. In addition, Anopheles bancroftii $(\mathrm{n}=30)$, Anopheles longirostris $(\mathrm{n}=2)$, An. punctulatus $(\mathrm{n}=3)$, Aedes $\mathrm{spp}(\mathrm{n}=54)$, Culex spp $(\mathrm{n}=42)$, Mansonia spp $(\mathrm{n}=2)$ and Armigeres spp $(\mathrm{n}=5)$ were collected (Table 1$)$.

The mean number of $A n$. farauti collected per $10 \mathrm{~m}$ barrier screen per night was 7.8 (Table 1). By abdominal status these An. farauti were: $59.2 \%$ blood fed $(\mathrm{n}=184)$, $40.2 \%$ unfed $(\mathrm{n}=125)$ and $0.6 \%$ gravid $(\mathrm{n}=2)$ (Table 1$)$. The mean nightly, human landing rate for An. farauti during the same period was $20.6 / \mathrm{p} / \mathrm{n}(\mathrm{n}=412)$ with the major landing collection peak occurring between $1900 \mathrm{~h}$ and $2000 \mathrm{~h}$ and a secondary peak between $2300 \mathrm{~h}$ and midnight (Figure 3C). Peak collection time for resting mosquitoes on the barrier screen was between midnight and $0100 \mathrm{~h}$ (Figure 3D). Anopheles bancroftii, An. longirostris and An. punctulatus were not captured in landing catches during the nights when collections on the barrier screens were undertaken.
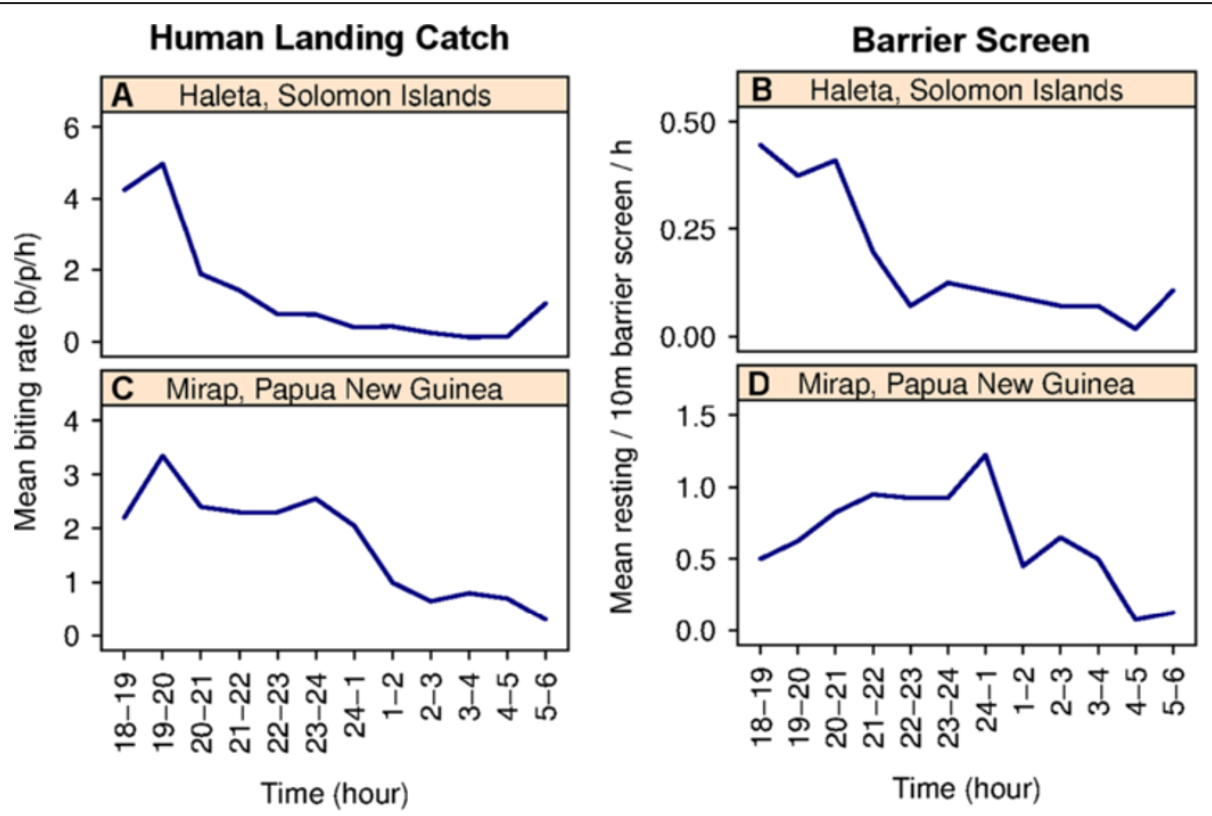

Figure 3 Comparison of the mean densities of Anopheles farauti captured with human landing catches and resting on the barrier screen in the Solomon Islands (A, B, respectively) and Papua New Guinea (C, D, respectively). 


\section{Discussion}

The hypothesis that a barrier screen trap can detain mosquitoes sufficiently to allow their collection was validated in all three countries where trialled. The barrier screen was specifically designed for collecting an unbiased sample of outdoor resting mosquitoes to provide specimens for calculating the human blood index. This novel tool could directly replace unproductive and/or biased searches for mosquitoes resting outdoors in vegetation, indoors or in outdoor shelters. The use of barrier screens was more time-effective as a means of collecting resting, blood-engorged members of the An. punctulatus group, when population densities are low, compared to searching natural resting sites. Previous studies in Mebat village, PNG, in which the bush was searched for resting anophelines, found 27 engorged members of the An. punctulatus group during 128 days of searching when the mean nightly human landing rate was $8 / \mathrm{p} / \mathrm{n}$ [18]. In the same study, only 12 engorged anophelines were collected in Hudini village during 128 search days despite a mean nightly human landing rate of $85 / \mathrm{p} / \mathrm{n}$. The use of a barrier screen as a sampling tool for resting mosquitoes was more time effective: in the Solomon Islands over a 14-day period, 70 blood-fed $A n$. farauti were collected even though the human landing rate during this period was only $16.5 / \mathrm{p} / \mathrm{n}$; in PNG, 184 engorged $A n$. farauti were captured on the barrier screen in a 10-day period when the human landing catch averaged $20.6 / \mathrm{p} / \mathrm{n}$.

Positioning the barrier screen between the hosts and oviposition/resting sites enabled samples of blood-fed mosquitoes to be collected to estimate the HBI as well as to sample mosquitoes questing for blood meals. In Indonesia analyses of the limited numbers of blood meals in anophelines confirmed previous descriptions of An. vagus and An. sundaicus as zoophilic [3] as human blood was not identified in any anopheline collected on the barrier screen. Although only a very limited number of blood-fed anophelines were collected in Indonesia, the results were sufficiently encouraging to continue the experiments in the Solomon Islands and PNG. Blood meal analyses were conducted on An. farauti collected in the Solomon Islands, and at this study site the HBI was very high. Previous literature indicated that An. farauti blood-feeding habits vary from zoophilic to anthropophilic [13,16-22,29]. The high HBI recorded in this study may be a function of the relative abundance of humans compared to domestic animals (human, pig, and dog populations in the vicinity of the barrier screen numbered 63, 11 and 4, respectively) and/or an innate preference for feeding on humans.

The density of An. farauti captured on the barrier screen paralleled the results of human landing catches with most resting An. farauti captured between $1800 \mathrm{~h}$ and $2100 \mathrm{~h}$ in the Solomon Islands and before midnight in PNG. These results, coupled with anecdotal observations made during the course of the experiments indicate that the barrier screen does indeed act as a barrier which temporarily detains mosquitoes, opposed to being an artificial resting site. Notably, greater numbers of resting $A n$. farauti captured on the village side of the barrier screen may reflect the willingness of blood-fed $A n$. farauti to rest longer on the barrier screen than mosquitoes questing for blood. Such questing mosquitoes would be more likely to encounter the barrier screen on the oviposition site side but would be expected to fly over or around the barrier screen to continue following cues to the locations of potential blood meals in the village. The success of the barrier screen as a tool for collecting blood-fed mosquitoes provides the baseline for designing detailed experiments to systematically observe individual mosquito resting behaviour as well as to study the population-level feeding behaviour. Of note here that is the observation that most mosquitoes, and particularly anophelines, rest near the ground is consistent with both the observations in The Gambia of Giglioli [9] for Anopheles melas and Damar et al. [30] in Indonesia who reported that Anopheles aconitus, Anopheles subpictus and Anopheles indefinitus rest indoors at a median height of $38 \mathrm{~cm}$ above the floor.

Although the experiments were designed to sample female anophelines, the barrier screen also useful for sampling culicine species, with $>1,500$ specimens of two Culex species being captured during seven nights in Indonesia. In addition, a small number of male Anopheles and Aedes spp were also collected on the barrier screens. Interestingly, most male Aedes were captured on the barrier screen between $0200 \mathrm{~h}$ and $0500 \mathrm{~h}$. With further refinements, screens or similar barriers may provide a simple method for sampling the male mosquito population, an area in which far too little is presently known.

The potential use of novel barrier screens as a method for sampling blood-fed mosquitoes seeking resting sites, female mosquitoes questing for blood meals and male mosquitoes, was evaluated in three countries that are well known for their exophilic and exophagic anophelines. Importantly, the technique is largely free of the biases associated with solely collecting mosquitoes resting inside households [2] and is much more effective than searching for outdoor-resting mosquitoes in the natural vegetation. The approach was validated as a means of sampling engorged females for HBI determination and shows promise as an approach to sampling host-seeking female mosquitoes as well as male mosquito populations. It is proposed that barrier screens could replace direct searching of vegetation for collecting outdoor-resting anophelines in many locations. 


\section{Conclusions}

Barrier screens placed to intercept mosquitoes between blood feeding, resting and ovipositing provide a novel way to sample mosquitoes. These barrier screens are easy and economical to construct and are effective in capturing Anopheles, Culex and Aedes spp including both males and females. Manipulating the locations where barrier screens are placed provides opportunities for capturing mosquitoes to better understand when and where mosquitoes move between host-seeking, egg laying and resting activities.

\section{Competing interests}

The authors declare that they have no competing interests.

\section{Authors' contributions}

TRB conceived the concept and instituted the initial experimental designs. TLR, LJR and SS supervised the overall field studies in the Solomon Islands, PNG and Indonesia, respectively. $\mathrm{HB}, \mathrm{NWB}$ and RDC contributed to the experimental designs and conducted the field studies in the Solomon Islands. FHC and NFL contributed to the experimental designs and coordinated the studies in the Solomon Islands and Indonesia countries. All authors read and approved the final manuscript.

\section{Acknowledgements}

This work was supported by Grant No. 45114 from the Bill and Melinda Gates Foundation to the Malaria Transmission Consortium for the work in Indonesia and the Solomon Islands. In addition, the support of the National Institutes of Health for the International Center of Excellence in Malaria Research in the Pacific (subawards to James Cook University and the Papua New Guinea Institute of Medical Research; award number SU19AI08986-03) is gratefully acknowledged. Technical assistance when conducting the field collections was provided by Allen Apairamo and John Lodo of the Vector Borne Disease Control Programme, Solomon Islands and Gussy Koimbu and Michelle Katusele of Papua New Guinea Institute of Medical Research. The analysis of blood meal contents with ELISA and PCRs were conducted by Dr Brandyce St. Laurent at the University of Notre Dame, Capt Weng Chow at $\mathrm{AMI}$ and Peter Moore at UQ. The authors thank the communities of each study village for their support as well as the support of Albino Bobogare, Director of National Vector Borne Disease Control Programme, Solomon Islands, and Professor Peter Siba, Director of the Papua New Guinea Institute of Medical Research.

\section{Author details}

'James Cook University, Queensland Tropical Health Alliance, Cairns, QLD 4870, Australia. ${ }^{2}$ Centers for Disease Control and Prevention, Division of Parasitic Diseases and Malaria, Atlanta, GA 30333, USA. ${ }^{3}$ Papua New Guinea Institute of Medical Research, Madang, Papua New Guinea. ${ }^{4}$ Case Western Reserve University, Cleveland, OH 44106, USA. ${ }^{5}$ National Vector Borne Disease Control Programme, Ministry of Health, Honiara, Solomon Islands. ${ }^{6}$ School of Biological Sciences, University of Queensland, St. Lucia, QLD 4068, Australia. ${ }^{7}$ CSIRO Ecosystem Sciences, Dutton Park, Brisbane, QLD 4102, Australia. ${ }^{8}$ Australian Army Malaria Institute, Gallipoli Barracks, Enoggera, QLD 4051, Australia. ${ }^{9}$ National Institute of Health, Research and Development, Health Ecology Research and Development Center, Jakarta, Indonesia. ${ }^{10}$ Eck Institute for Global Health, Department of Biological Sciences, University of Notre Dame, Notre Dame, IN 46556, USA.

Received: 5 December 2012 Accepted: 31 January 2013 Published: 5 February 2013

\section{References}

1. World Health Organization: Terminology of malaria and malaria eradication. In Monograph Series No 13. Geneva: World Health Organization; 1963.

2. Garrett-Jones C: The human blood index of malaria vectors in relation to epidemiological assessment. Bull World Health Organ 1964, 30:241-261.

3. Sinka M, Bangs M, Manguin S, Chareonviriyaphap T, Patil A, Temperley W, Gething P, Elyazar I, Kabaria C, Harbach R, Hay S: The dominant Anopheles vectors of human malaria in the Asia-Pacific region: occurrence data, distribution maps and bionomic precis. Parasit Vectors 2011, 4:89.

4. Sinka M, Bangs M, Manguin S, Coetzee M, Mbogo C, Hemingway J, Patil A, Temperley W, Gething P, Kabaria C, Okara R, Van Boeckel T, Godfray HC, Harbach R, Hay S: The dominant Anopheles vectors of human malaria in Africa, Europe and the Middle East: occurrence data, distribution maps and bionomic precis. Parasit Vectors 2010, 3:117.

5. Sinka M, Rubio-Palis $Y$, Manguin S, Patil A, Temperley W, Gething P, Van Boeckel T, Kabaria C, Harbach R, Hay S: The dominant Anopheles vectors of human malaria in the Americas: occurrence data, distribution maps and bionomic precis. Parasit Vectors 2010, 3:72.

6. Silver JB: Mosquito ecology: field sampling methods. 3rd edition. New York: Springer; 2008

7. Sikulu M, Govella N, Ogoma S, Mpangile J, Kambi S, Kannady K, Chaki P, Mukabana W, Killeen G: Comparative evaluation of the Ifakara tent trap-B, the standardized resting boxes and the human landing catch for sampling malaria vectors and other mosquitoes in urban Dar es Salaam, Tanzania. Malar J 2009, 8:197.

8. Odiere M, Bayoh MN, Gimmnig JE, Vulule JM, Irungu LW, Walker ED: Sampling outdoor, resting Anopheles gambiae and other mosquitoes (Diptera: Culicidae) in Western Kenya with clay pots. J Med Entomol 2007, 44:14-22.

9. Giglioli G: The influence of irregularities in the bush perimeter of the cleared agricultural belt around a Gambian village on the flight range and direction of approach of a population of Anopheles gambiae melas. In Proceedings of the XIlth International Congress of Entomology. London: Royal Entomological Society of London; 1964:757-758. 8-16 July.

10. Gillies MT, Wilkes TJ: The effect of high fences on the dispersal of some West African mosquitoes (Diptera: Culicidae). Bull Entomol Res 1978, 68:401-408

11. Belkin JN: The mosquitoes of the South Pacific (Diptera, Culicidae). Berkeley and Los Angeles: University of California Press; 1962.

12. Beebe NW, Cooper RD: Distribution and evolution of the Anopheles punctulatus group (Diptera: Culicidae) in Australia and Papua New Guinea. Int J Parasitol 2002, 32:563-574.

13. Burkot TR, Graves PM, Paru R, Lagog M: Mixed blood feeding by the malaria vectors in the Anopheles punctulatus complex (Diptera: Culicidae). J Med Entomol 1988, 25:205-213.

14. Avery JG: The epidemiology of disappearing malaria in the Solomon Islands, PhD Thesis. Sheffield: University of Sheffield; 1977.

15. Chow CY: Bionomics of malaria vectors in the Western Pacific region. Southeast Asian J Trop Med Publ Health 1970, 1:40-57.

16. Bruce-Chwatt LJ, Garrett-Jones C, Weitz B: Ten years' study (1955-64) of host selection by anopheline mosquitos. Bull World Health Organ 1966, 35:405-439.

17. Afifi SE, Spencer M, Hudson PB, Tavil NW: Biting prevalence and malaria transmission patterns in the Anopheles punctulatus complex (Diptera: Culicidae) in Papua New Guinea. Aust J Exp Biol Med 1980, 58:1-17.

18. Burkot TR, Dye C, Graves PM: An analysis of some factors determining the sporozoite rates, human blood indicies, and biting rates of members of the Anopheles punctulatus complex in Papua New Guinea. Am J Trop Med Hyg 1989, 40:229-234.

19. Charlwood JD, Dagoro H, Paru R: Blood-feeding and resting behaviour in the Anopheles punctulatus Dönitz complex (Diptera: Culicidae) from coastal Papua New Guinea. Bull Entomol Res 1985, 75:463-476.

20. Graves PM, Burkot TR, Saul A, Hayes RJ, Carter R: Estimation of Anopheline survival rate, vectoral capacity and mosquito infection probability from malaria vector infection rates in villages near Madang, Papua New Guinea. J Appl Ecol 1990, 27:124-147.

21. Hii JLK, Mai A, Mellor S, Lewis D, Alexander N, Alpers M: Spatial and temporal variation in abundance of Anopheles (Diptera: Culicidae) in a malaria endemic area in Papua New Guinea. J Med Entomol 1997, 34:193-205.

22. Spencer M: Blood preferences of Anopheles farauti. P N G Med J 1964, 7:19.

23. Zahiri NS, Su T, Mulla MS: Strategies for the management of resistance in mosquitoes to the microbial control agent Bacillus sphaericus. J Med Entomol 2002, 39:513-520.

24. O'Connor C, Soepanta A: Illustrated key to female anophelines of Indonesia. Jakarta, Indonesia: Directorate of Communicable Disease, $\mathrm{MoH}$ and US Naval Medical Research; 1989. 
25. Lee DJ, Hicks MM, Griffiths M, Debenham ML, Bryan JH, Russell RC, Geary M, Marks EN: The culicidae of the Australasian region, Volume 5. Canberra: Australian Government Publishing Service; 1987.

26. Beebe NW, Saul A: Discrimination of all members of the Anopheles punctulatus complex by polymerase chain reaction - restriction fragment length polymorphism analysis. Am J Trop Med Hyg 1995, 53:478-481.

27. Beebe NW, Foley DH, Cooper RD, Bryan JH, Saul A: DNA probes for the Anopheles punctulatus complex. Am J Trop Med Hyg 1996, 54:395-398.

28. Kent RJ, Norris DE: Identification of mammalian blood meals in mosquitoes by a multiplexed Polymerase Chain Reaction targeting Cytochrome B. Am J Trop Med Hyg 2005, 73:336-342.

29. Chow CY: Ecology of malaria vectors in the Pacific. Cah ORSTOM ser Ent med et Parasitol 1969, 7:93-97.

30. Damar T, Fleming GA, Gandahusada S, Bang YH: Nocturnal indoor resting heights of the malaria vector Anopheles aconitus and other anophelines (Diptera: Culicidae) in Central Java, Indonesia. J Med Entomol 1981, 18:362-365.

doi:10.1186/1475-2875-12-49

Cite this article as: Burkot et al:: Barrier screens: a method to sample blood-fed and host-seeking exophilic mosquitoes. Malaria Journal 2013 12:49.

\section{Submit your next manuscript to BioMed Central and take full advantage of:}

- Convenient online submission

- Thorough peer review

- No space constraints or color figure charges

- Immediate publication on acceptance

- Inclusion in PubMed, CAS, Scopus and Google Scholar

- Research which is freely available for redistribution 